Mid-life approaches to prevention of ill health in later life

Louise Lafortune, PhD; Steven Martin, MPhil; Sarah Kelly, PhD

Corresponding Author: Louise Lafortune, PhD

Institute of Public Health, Forvie Site

University of Cambridge School of Clinical Medicine

Box 113 Cambridge Biomedical Campus, Cambridge, CB2 OSR

+44 (0)1223 746556

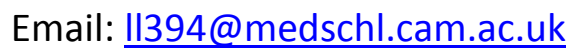

\title{
Co-author Affiliations:
}

- Sarah Kelly, PhD; Institute of Public Health, University of Cambridge School of Clinical

Medicine, Cambridge, UK. Email: sak65@medschl.cam.ac.uk

- Steven Martin, MPhil; Institute of Public Health, University of Cambridge School of Clinical Medicine, Cambridge, UK. Email: steamartin@riseup.net

Word count: 1000

Revision date: 21 November 2016 


\section{Source of Review}

The Cambridge Institute of Public Health conducted three rapid evidence reviews to inform the National Institute for Health and Care Excellence (NICE) guideline on mid-life approaches to prevent or delay onset of dementia, disability and frailty (DDF) published on 20 October, $2015 .^{1}$ The full version of the guideline and its supporting documents contain details of the methods used to derive the recommendations (www.nice.org.uk/guidance/ng16). The effectiveness of individually directed mid-life (40-64 years) interventions to increase the uptake and maintenance of healthy behaviours are summarized here. No studies reported on the effect of midlife interventions on DDF outcomes. ${ }^{2}$

\section{Background}

There is growing evidence of the association between low physical activity, smoking, alcohol consumption, poor diet, and ill health in later life. ${ }^{3}$ Public health guidance provides tailored recommendations to promote healthy behaviours in children, the general adult population, and to some extent in older adults. Yet, few recommendations target mid-life when these behaviours tend to become entrenched. Individuals in mid-life are highly heterogeneous but share some of the same issues when it comes to changing or maintaining healthy behaviours. ${ }^{4}$ It should thus be possible to effectively have adults in mid-life engage in health promoting activities with approaches tailored to their particular needs and circumstances. This is the aim of the NICE guideline on mid-life interventions. ${ }^{1}$

\section{Summary of Findings}

The review identified 30 systematic reviews and 47 primary intervention studies aimed at promoting uptake and maintenance of healthy behaviours specifically in mid-life. ${ }^{2}$ The evidence 
for individually directed interventions aimed at physical activity, diet, smoking, alcohol consumption, and multiple risk behaviours showed significant uptake of healthy behaviours with some evidence of cost-effectiveness and no evidence of adverse events (Table 1). No studies targeting social, leisure and cognitive activities, or hearing and sight behaviours were identified.

\section{Limitations on the Evidence}

This rapid review focuses on individually directed behavioural interventions, and exclude interventions targeting environmental cues, which we know drive our behaviours. ${ }^{6}$ The search strategies (i.e. filtered for mid-life-related terms in the title, abstract or related MeSH indexing) will not have identified relevant studies that have recruited only/mainly individuals in mid-life without specifying mid-life terms. Short follow-up periods mean we don't know if and how long changes in behaviours are maintained after the intervention stops, and small sample sizes compromise the power to detect small changes in behaviour. Due to different choices of control groups and ways of measuring behavioural risk factors and outcomes, we can't combine findings to assess whether one type of intervention is better than another. Finally, most studies used selfreported measures of behaviour, which may result in overestimated effect sizes because these often don't reflect actual behaviours.

\section{$\underline{\text { Implications for Practice }}$}

Generally, interventions providing detail information to promote specific behaviours and highlight their immediate and long-term benefit can improve someone's health and discourage behaviours that can lead to poor health. This includes explaining how, at any age, modest increases in physical activity as part of everyday life and maintaining a healthy diet and body weight can be beneficial; encouraging smokers to stop smoking; explaining that alcohol consumption can increase the risk 
of DDF and encouraging people to reduce the amount they drink as much as possible.

Specifically, structured exercise programme (e.g. stretching, aerobic exercise, walking, weight training, cycling, yoga) provided as tailored individual or group support interventions can influence decisions to start and maintain physical activity. These can be provided as multimodal interventions ranging from a single motivational education session (these were less effective) to extensive supervised exercise sessions. Interventions that include support, self-monitoring, rewards, personal goal setting and/or provision of information about local opportunities are more likely to produce better outcomes.

For diet, there is some evidence that individually tailored advice, group programmes or printed dietary advice can be effective in improving dietary behaviour e.g. explaining that regularly consuming meals, snacks and beverages high in salt, fats and sugars is bad for health, and that a diet mainly based on vegetables, fruits, beans and pulses, wholegrains and fish, is likely to improve it.

For smoking, reduction interventions delivered by various methods (information leaflets, practice nurses, outreach workers, the internet, specialist smoking cessation clinic, and telephony), can significantly influence quitting intention, quit attempts and smoking cessation, and can be carried out with the aid of pre-quit nicotine replacement therapy. Interventions combining self-help, behavioural, counselling and/or educational components can change smoking attitudes and behaviours; patients can be given the choice of a variety of strategies (before quit day and quitting abruptly, with no prior reduction) to assist in behavioural change. 
Evidence shows the availability of alcohol does impact individual consumption levels so interventions aimed at making alcohol less accessible, affordable and acceptable should be implemented $^{1}$ in addition to brief and tailored information packages for minor problem drinking or psychotherapy for mild to moderate dependence.

Given that unhealthy behaviours tend to co-occur, lifestyle interventions such as cognitive behavioural therapy, motivational interviewing and problem solving, as well as education and counselling targeting more than one health behaviours (e.g. physical activity and diet) can be effective in improving multiple risk behaviours.

Across risk behaviours interventions can be delivered face-to-face or internet-based but information about their comparative effectiveness and cost-effectiveness is limited; as well as for minority groups.

\section{Conclusions}

Consistent evidence suggests that individually based modification of behavioural risks can occur in mid-life, and can be cost-effective ${ }^{5}$ if these changes are sustained in the long term. This requires interventions designed to foster long-term maintenance of healthy behaviours as well as a good understanding of individual and environmental barriers and facilitators to behaviour change in midlife and how those evolve as people reach retirement and older age. ${ }^{7}$ Understanding what types and modalities of interventions work for older populations ${ }^{8}$ combined with evidence from longer-term studies with validated outcomes measures are also needed to establish the extent to which these changes - or a combination thereof - impact late life outcomes. Finally, real-life evaluations accounting for the sociocultural and environmental factors that influence the 
effectiveness of interventions are urgently needed to deliver approaches tailored to particular context and populations. 


\section{REFERENCES}

1. NICE Dementia, disability and frailty in later life - mid-life approaches to delay or prevent onset. NICE guideline, published 20 October 2015. Nice.org.uk/guidance/ng16. Accessed March 12, 2016.

2. Lafortune L, Martin S, Kelly S, Kuhn I, Cowan A, Brayne C. Effectiveness and cost-effectiveness of mid-life interventions for increasing the uptake and maintenance of healthy lifestyle behaviours and the prevention or delay of dementia, disability, frailty and non-communicable chronic diseases related to modifiable lifestyle risk factors. NICE guideline 16, supporting evidence. NICE Final Report. 2014. https://www.nice.org.uk/guidance/ng16/evidence/evidence-review-3552743969. Accessed March 12, 2016

3. Lafortune L, Martin S, Kelly S, Kuhn I, Remes O, Cowan A, Brayne C. Behavioural risk factors in mid-life associated with successful ageing, disability, dementia and frailty in later life: a rapid systematic review. PLoS ONE. 2016; 11(2):e0144405. doi:10.1371/journal.pone.0144405.

4. Kelly S, Martin S, Kuhn I, Cowan A, Brayne C, Lafortune L. Barriers and Facilitators to the Uptake and Maintenance of Healthy Behaviours by People at Mid-Life: A Rapid Systematic Review. PLoS ONE. 2016; 11(1):e0145074. doi:10.1371/journal. pone.0145074.

5. Van Baal P, Hoogendoorn M, Fisher A. Preventing dementia by promoting physical activity and the long-term impact on health and social expenditures. Preventive Medicine. 2016; 85:78-83. doi:10.1016/j.ypmed.2016.01.013. 
6. Hollands GJ, Marteau TM, Fletcher P. Non- conscious processes in changing health-related behaviour: a conceptual analysis and framework. Health Psychology Review. 2016 DOI:

$10.1080 / 17437199.2015 .1138093$

7. Kelly S, Lafortune L, Martin S, Smailagic N, Cowan A, Brayne C. A systematic review of issues (barriers and facilitators) that prevent or limit or that help and motivate the uptake and maintenance of healthy behaviours in older people. PROSPERO 2014:CRD42014015557 Available from http://www.crd.york.ac.uk/PROSPERO_REBRANDING/display_record.asp?ID=CRD42014015557

8. Lafortune L, Kelly S, Martin S, Smailagic N, Cowan A, Brayne C. A systematic review of interventions in older age for increasing the uptake and maintenance of healthy behaviours that may impact on successful ageing. PROSPERO 2014:CRD42014015554 Available from http://www.crd.york.ac.uk/PROSPERO_REBRANDING/display_record.asp?ID=CRD42014015554 
ARTICLE INFORMATION

Funding/Support: This work was funded by the National Institute for Health and Care Excellence (NICE), invitation to tender reference DDER 42013, and supported by the National Institute for Health Research School for Public Health Research (NIHR SPHR) Ageing Well Programme.

Role of the Funder/Sponsor: The scope of the work was defined by NICE and the protocol was agreed with NICE prior to the start of work. The funders had no role in data analysis, preparation of the manuscript or decision to publish.

Additional Contributions: The authors acknowledge Andy Cowan (University of Cambridge, Institute of Public Health) for his support with data and report preparation; Isla Kuhn (University of Cambridge Medical Library) for her support with the literature searches; Professor Carol Brayne for her input throughout the project. The author would like to thank Hilary Chatterton, Catherine Swann, Hugo Crombie and Paul Levay from NICE and all members of the Public Health Advisory Committee for their useful suggestions and constructive comments on the design of the reviews. 
- Behavioural interventions appear to be more effective than cognitive interventions that target knowledge, attitudes, or beliefs

- Motivational PA interventions can increase PA behaviour and decrease sedentary behaviour

- Interventions such as structured exercise programmes, yoga and walking can influence decisions to start and maintain PA

- The most effective interventions offer tailored individual and group support; information about local opportunities for engaging in PA

- Evidence for the effect of individual supportive and educational interventions on PA behaviours is inconclusive

Internet-based PA interventions produced small to moderate increase in PA behaviour at 12 months

- Screening for baseline PA levels and including educational components in the intervention significantly increased effectiveness

- Evidence is inconclusive whether face-to-face PA interventions are more/less effective than remote and web 2.0 approaches

Mass media campaigns may increase awareness and promote walking but may not lead to recommended PA levels

- Campaigns promoting PA as 'social norm' may reduce sedentary behaviour but are less effective than other strategies to increase PA

\section{DIET}

- Printed dietary feedback tailored to individual dietary intake and delivered over 3 months is more effective to improve fruit intake than either two dietician-led small group nutrition education sessions or a waiting list control

- Intensive behavioural modification program can change diet in postmenopausal women with high dietary fat intakes (at 6yrs)

\section{SMOKING}

Cessation interventions can significantly influence quitting intention, quit attempts, and quit rates

- Reducing cigarettes smoked before quit day and quitting abruptly with no prior reduction produced comparable quit rates

- Cessation interventions delivered in Hispanic adults can reduce smoking rates in the short term; long term effects are uncertain

- Telephone-based cessation interventions (counselling calls; quit lines) are cost-effective based on conventional standards

\section{ALCOHOL}

Internet-based self-help and therapy can both change alcohol consumption in the short term for problematic alcohol users

- Internet-based therapy may offer better value for money compared to internet-based self-help

- Nurse-led intervention (five-ten minutes face-to-face screening and brief intervention using the DrinkLess protocol) had no effect on excessive alcohol consumption over standard advice delivered by nurses in primary health care

- Availability of a system-level reminder aimed at clinicians to facilitate brief interventions did not by itself result in substantial use of the clinical reminder nor in resolution of unhealthy drinking

\section{MULTICOMPONENT}

- Combined diet and exercise modification can lead to significant improvements in nutrition (i.e. decrease in dietary fat intake)

- Internet-based lifestyle interventions that provide tailored or generic information can improve PA, diet and weight loss behaviours interventions consisting of more than one component may be more effective than single component interventions

- Multicomponent interventions delivered via social networking sites are not effective in people aged over 50

- Cultural and gender specific cognitive behavioural interventions may increase PA and improve diet in African American women

3 SR $[++][++][+] ; 3$ PS $[++][++][+]$

- $1 \mathrm{SR}[+]$

- 2 PS $[++][++] ; 4$ PS $[+]$

- 4 PS $[++][++][+][-]$

- $1 \mathrm{SR}[++]$

- 1 PS [++]; 2 PS [+]

$2 \mathrm{SR}[++][+]$

- $2 \mathrm{SR}[++][+]$

- 2 SR $[++][+]$

3 SR $[++][+][-]$

- $2 \mathrm{SR}[+]$

- 1 PS [+]

- 1 PS $[++]$

5 PS [++]; 1 PS [+]

- $1 \mathrm{SR}[++]$

- 1 SR [-]

- 1 PS [+]; 1 model [+]

\section{PS [++]; 1 PS [++]}

- $1 \mathrm{PS}[++]$

- 1 PS [++]

- 1 PS [++]

- 2 PS [+][++]

- $1 \mathrm{SR}[+]$

- $1 \mathrm{SR}[+]$

- 1 PS [-] 
SR: Systematic review focused on mid-life OR with included studies conducted predominantly in mid-life; PS: Primary intervention study focused on mid-life. [++] High quality SR or PS; [+] Moderate quality SR or PS; [-] Low quality and/or high risk of bias SR or PS. 